\title{
Elaboração e validação de diretrizes de educação em saúde para o ensino médio
}

\author{
Elaboration and validation of health education guidelines for high school \\ Elaboración y validación de directrices de educación en salud para la enseñanza media \\ Icaro Kleysson de Souza Carvalho"* Luciana Marques Andreto².
}

\begin{abstract}
RESUMO
Objetivo: Elaborar e validar diretrizes de Educação em Saúde para estudantes do Ensino Médio. Métodos: Trata-se de estudo metodológico de elaboração e validação. A primeira etapa consistiu na elaboração de diretrizes de Educação em Saúde para o Ensino Médio do Instituto Federal de Educação do Sertão Pernambucano. A segunda consistiu na validação das diretrizes por 15 juízes para verificar o nível de concordância das afirmações. $E$ a terceira, consistiu na validação semântica das diretrizes. O método Delphi foi utilizado para validação. Resultados: na primeira etapa do estudo foram construídos 79 itens distribuídos em nove eixos. $\mathrm{Na}$ etapa de validação de conteúdo, 45 desses itens, distribuídos em oito eixos, foram validados pelos juízes. O Eixo Saúde Bucal não foi validado. Nesta etapa, o instrumento Diretrizes de Educação em Saúde para o Ensino Médio foi validado com 91\% de concordância dos Juízes especialistas. $\mathrm{Na}$ última etapa de validação semântica, a população alvo analisou os aspectos semânticos do instrumento e validaram com 96\% de consenso. Conclusão: Este instrumento pode tornar a prática e ações dos profissionais de saúde dos Institutos Federais de Educação, no planejamento da Educação em Saúde, mais segura e com base científica.
\end{abstract}

Palavras-chave: Educação em saúde, Serviços de saúde escolar, Estudos de validação, Diretrizes.

\begin{abstract}
Objective: Develop and validate health education guidelines for high school students. Methods: This is a methodological study of elaboration and validation. The first stage consisted of the elaboration of health education guidelines for high school at the Federal Institute of Education of Sertão Pernambucano. The second consisted of the validation of the guidelines by 15 judges to verify the level of agreement of the statements. And the third, consisted of the semantic validation of the guidelines. The Delphi method was used for validation. Results: in the first stage of the study, 79 items were constructed, distributed over nine axes. In the content validation stage, 45 of these items, distributed in eight axes, were validated by the judges. The Oral Health Axis has not been validated. At this stage, the instrument of Health Education Guidelines for High School was validated with $91 \%$ agreement by law experts. In the last stage of semantic validation, the target population analyzes the semantic aspects of the instrument and is validated with a $96 \%$ consensus. Conclusion: This instrument can make the practices and actions of health professionals at Federal Institutes of Education, in Health Education planning, safer and scientifically based.
\end{abstract}

Keywords: Health education, School health services, Validation studies, Guidelines.

\footnotetext{
1 Instituto Federal de Educação, Ciência e Tecnologia Sertão Pernambucano (IFSertão - PE)

Serra Talhada - PE. *E-mal: Icaro.Carvalho@ifsertao-pe.edu.br

2 Faculdade Pernambucana de Saúde (FPS), Recife - PE.
} 


\section{RESUMEN}

Objetivo: Desarrollar y validar las pautas de Educación para la Salud para estudiantes de secundaria. Métodos: estudio metodológico de elaboración y validación. La primera etapa consiste en la elaboración de directrices educativas para la salud de la educación secundaria en el Instituto Federal de Educación de Sertão Pernambucano. El segundo consistió en la validación de las directrices por 15 jueces para verificar el nivel de acuerdo de las declaraciones. Y el tercero, es la validación semántica de las pautas. El método Delphi se utilizó para la validación. Resultados: en la primera etapa del estudio, se construyeron 79 artículos, distribuidos en pequeñas cantidades. En la etapa de validación de contenido, 45 de estos ítems, distribuidos entre ellos, fueron validados por los jueces. El Eje de Salud Oral no ha sido validado. En esta etapa, los expertos expertos validaron las directrices para la educación en salud para el instrumento de la escuela secundaria con una precisión del $91 \%$. En la última etapa de la validación semántica, el objetivo era analizar los aspectos semánticos del instrumento y validarlo con un consenso del $96 \%$. Conclusión: Este instrumento podría proporcionar la práctica y las acciones de los profesionales de la salud en los Institutos Federales de Educación, en la planificación de la Educación para la Salud, de manera más segura y con una base científica.

Palabras clave: Educación en salud, Servicios de salud escolar, Estudios de validación, Pautas.

\section{INTRODUÇÃO}

A Educação em Saúde mostra-se como um importante meio de assistir o estudante e a relevância dessa temática foi ratificada pelo Governo Brasileiro e por políticas e programas internacionais através da criação, execução e investimentos nas Escolas Promotoras de Saúde, no Programa Saúde na Escola (PSE), além de estar presente em projetos e artigos científicos que versam sobre a Educação em Saúde no contexto escolar (ROGERS R, et al., 2017; GUETERRES ÉC, et al., 2017; MCISAAC JLD, et al., 2016).

No âmbito mundial, após a criação da Rede Latino-Americana de Escolas Promotoras de Saúde durante o Congresso de Saúde Escolar no Chile, em 1995, ocorreram importantes sistematizações e direcionamentos na Educação em saúde no contexto escolar. A estratégia da criação dessa rede representa a opção para a institucionalização e conformação de políticas públicas de saúde escolar incentivada pela Organização PanAmericana de Saúde (MONT'ALVERNE DGB e CATRIB AMF, 2013; CARDOSO V, et al., 2008).

Escolas promotoras de saúde são estratégias de promoção da saúde no âmbito escolar e um mecanismo articulado de esforços e recursos multissetoriais, focado nas condições de saúde e bem-estar, ampliando assim as oportunidades para um aprendizado de qualidade e o desenvolvimento humano sustentável, para todos os integrantes das comunidades educativas (CARDOSO V, et al., 2008).

No Brasil, em 1971, a Lei número 5.692, revogada pela Lei no 9.394 de 20 de dezembro de 1996 que estabelece as diretrizes e bases da educação nacional veio introduzir formalmente, no ambiente escolar, a temática da saúde. Em paralelo ao currículo formal escolar, a programação dos temas relacionados à saúde deveria ser trabalhada não no formato de disciplina, mas de forma continuada (PARÂMETROS CURRICULARES NACIONAIS SAÚDE, 1997).

Nacionalmente, o Programa Saúde na Escola tem como finalidade contribuir para a formação integral dos estudantes da rede pública de educação básica por meio de ações de prevenção, promoção e atenção à saúde. Constitui estratégia para a integração e a articulação permanente entre as políticas e ações de educação e de saúde, com a participação da comunidade escolar, envolvendo as equipes de saúde da família e da educação básica (BRASIL, 2007).

Em 2007, foi criado o Programa de Restruturação e Expansão das Universidades Federais, entre seus objetivos, destaca-se a diminuição das taxas de evasão dos estudantes, para isso, seria necessário criar uma política de assistência estudantil voltada para esse propósito (BLEICHER T e OLIVEIRA RCN, 2016).

Nessa perspectiva, foi instituido o Programa Nacional de Assistência Estudantil que prevê a atenção a saúde em áreas que deverão ser desenvolvidas ações da assistência estudantil nas universidades e Institutos 
Federais de Educação (BRASIL, 2010). Os Institutos Federais de Educação, Ciência e Tecnologia (IFEs) são instituições de educação superior, básica e profissional, pluricurriculares e multicampi, especializados na oferta de educação profissional e tecnológica nas diferentes modalidades de ensino (INSTITUIÇÃO, 2019).

Mesmo com a criação do decreto do Programa Nacional de Assistência Estudantil, não houve um programa de capacitação dos técnico-administrativos em educação quanto aos mecanismos de consecução dos objetivos da política. Alguns profissionais de saúde dos institutos Federais de Educação ainda apresentam dificuldades de trabalhar Educação em Saúde no contexto escolar (BLEICHER T e OLIVEIRA $\mathrm{RCN}, 2016)$.

Uma vez, que a função de profissionais de saúde no espaço escolar é algo novo e incipiente na realidade brasileira, pesquisas levam a acreditar que as equipes de técnicos-administrativos em educação dos Institutos Federais de Educação e Universidades Federais não embasam suas ações por meio de pesquisas acadêmicas (BLEICHER T e OLIVEIRA RCN, 2016).

As instituições de ensino devem proporcionar uma formação voltada para o desenvolvimento integral do ser humano e fornecer igualdade de condições para que o estudante consiga permanecer até a conclusão do curso (MINISTÉRIO DA EDUCAÇÃO, 1997).

A elaboração e validação de diretrizes são consideradas práticas que fornece direção de alto nível e recursos dentro das jurisdições escolares. As diretrizes de educação em saúde para estudantes do Ensino Médio representam uma forma científica de promoção de saúde que contempla uma população específica do contexto escolar (JONES SE, et al., 2015).

Sendo assim, o objetivo desse estudo foi elaborar e validar diretrizes de educação em saúde para estudantes do ensino médio do Instituto Federal de Educação do Sertão Pernambucano como um instrumento para realizar educação em saúde nesse contexto escolar tornando a prática e ações dos profissionais de saúde nesse ambiente mais segura e com base científica.

\section{MÉTODOS}

Trata-se de estudo metodológico de elaboração e validação de diretrizes de educação em saúde para o Ensino Médio (SANTOS FC, 2016; ALEXANDRE NM e COLUCI MZ, 2011; PILATTI L, et al., 2010; LIKERTRA, 1932; BOND KS, et al., 2016).

As etapas de elaboração e validação ocorreram no período entre maio de 2018 a julho de 2019.

A primeira etapa consistiu na elaboração de diretrizes de educação em saúde voltada para o contexto escolar do Ensino Médio em um Instituto Federal de Educação. Para isso foram pesquisadas literaturas científicas entre o ano 2014 e 2018 que apresentaram os descritores "educação em saúde" e "serviços de saúde escolar" ou descritores similares nas seguintes bases de dados: ERIC, BIREME, EBSCO e portal periódicos da Capes.

Também foram pesquisados documentos oficiais nacionais do Ministério da Saúde e do Ministério da Educação, além de documentos oficiais do Instituto Federal de Educação, Ciência e Tecnologia Sertão Pernambucano como o relatório gestor (ano 2016 e 2017) e o relatório do primeiro fórum discente da assistência estudantil que apresentaram os temas "educação", "saúde escolar" e "educação em saúde no contexto escolar".

A segunda etapa, a validação de conteúdo utilizou-se o Método Delphi (BOND KS, et al., 2016). Nesta etapa foram selecionados de forma aleatória e proporcional dois Institutos Federais de Educação por região do Brasil, totalizando 10 Institutos e para a seleção dos juízes foram aplicados os critérios de elegibilidade: ser profissional de saúde atuante na promoção de educação em saúde nos Institutos Federais de Educação, ter no mínimo dois anos de atuação e possuir titulação de Mestrado ou Doutorado em Educação ou Ciências da Saúde. Participaram da validação de conteúdo 15 juízes.

Para compor o grupo de juízes para validação de conteúdo e o grupo de profissionais de saúde (populaçãoalvo) para a validação semântica, foi enviado um instrumento por correio eletrônico, contendo explicações 
quanto aos objetivos, riscos e benefícios desse estudo. Após a leitura e consentimento eletrônico do Termo de Consentimento Livre e Esclarecido (TCLE) foi iniciada a pesquisa. Cada conteúdo presente nas diretrizes de educação em saúde para o ensino médio foi avaliado segundo os critérios adaptados da escala de Likert: "não deve ser incluído" (1), "pouco importante" (2), "parcialmente importante" (3), "muito importante" (4) e "indispensável” (5) (LIKERT RA, 1932).

A validação semântica do conteúdo foi realizada por profissionais de Saúde do IFSertão-PE. Os profissionais de saúde responderam um instrumento contendo perguntas sobre o perfil acadêmico e profissional e apontaram o nível de compreensão dos conteúdos apresentados em uma escala de pontos variando de 1 a 5 , sendo os valores igual ou superior a 4 , a correta compreensão do item. Os profissionais de saúde analisaram os aspectos semânticos dos itens quanto a inteligibilidade, clareza, pertinência e aparência (SANTOS FC, 2016; ALEXANDRE NM e COLUCI MZ, 2011; PILATTI L, et al., 2010; LIKERTRA, 1932; BOND KS, et al., 2016). Para a análise do conteúdo e análise semântica, calculou-se o nível de concordância dos juízes e profissionais de saúde através do Índice de Validade de Conteúdo (IVC). Considerou-se o índice de aceitação do conteúdo igual ou superior a $80 \%$ como critério de decisão sobre a pertinência do item no instrumento, ou sua modificação (SANTOS FC, 2016; ALEXANDRE NM e COLUCI MZ, 2011).

Esta pesquisa foi aprovada pelo Comitê de Ética em Pesquisa da Faculdade Pernambucana de Saúde CEP-FPS sob o número de CAAE 01117618.0.0000.5569, número de parecer 3.033.534 e seguiu a resolução número 466/2012 do Conselho de Nacional de Saúde (BRASIL, 2012).

\section{RESULTADOS E DISCUSSÃO}

A pesquisa na literatura científica e documentos oficiais resultou em um instrumento contendo 79 itens distribuídos em nove eixos. Para o processo de validação, participaram 15 juízes especialistas profissionais de saúde com no mínimo 2 anos de atuação na promoção de educação em saúde nos Institutos Federais de Educação, Ciência e Tecnologia no ambto do Ensino Médio, Mestre e/ou Doutor em Educação ou Ciências da Saúde.

Foram seis enfermeiros, cinco psicólogos, dois odontólogos, um nutricionista e um assistente social. A média de idade foi de 35,8 anos $\pm 6,5$ anos. Quanto à qualificação profissional $66,7 \%$ (10) eram mestres e $33,3 \%$ (5) eram doutores. Ressalta-se que 33,3\% (5), eram profissionais de saúde da região nordeste; $20 \%$ (3), da região norte; $20 \%$ (3), da região sul; 13,3\% (2), da região sudeste e 13,3\% (2), da região centro-oeste.

Na Tabela 1, observa-se que 38 conteúdos apresentaram índice de validade com concordância maior ou igual a 0,80 (IVC $\geq 0,80$ ). Nesta rodada, foi sugerida a reformulação de dois itens: reeducação alimentar para educação alimentar e nutricional e para o tema alergias alimentares para transtornos alimentares.

Ainda como sugestão, os juízes solicitaram a inclusão dos temas: desperdícios de alimentos, atividades físicas (alongamentos, caminhada e esportes), doença periodontal, bruxismo, halitose, câncer de boca, escalpelamento, doenças transmitidas por alimentos, terapias complementares (yoga, meditação, reiki e florais) e controle do estresse.

A Tabela 2 observou-se que do total de 52 conteúdos (novos itens e itens que não obtiveram consenso na primeira rodada do método), sete itens apresentaram IVC maior ou igual a 0,80 (IVC $\geq 0,80)$. Os itens que obtiveram IVC $<0,80$ foram excluídos e os itens que apresentaram IVC $\geq 0,80$ permaneceram, compondo os conteúdos das Diretrizes de Educação em Saúde para o Ensino Médio. Nesta segunda rodada, teve a participação de todos os juízes que participaram da primeira rodada e entre os conteúdos que tiveram IVC $\geq 0,80$, o item atividades físicas (alongamentos, caminhada e esportes) adicionado na primeira rodada apresentou índice de validade com concordância unanime (IVC=1,00).

Percebe-se que na Tabela 3 abaixo, todos os itens do Eixo sexualidade e reprodução foram avaliados como indispensável por $100 \%$ dos juízes. Além desses, os itens saúde corporal, saúde mental, violência, ansiedade, direitos humanos, inclusão e diversidade vacinas e atividades físicas (alongamentos, caminhada e esportes) também tiveram avaliação de $100 \%$ dos juízes como itens indispensáveis para compor as Diretrizes de Educação em Saúde para o Ensino Médio. 


\section{Revista Eletrônica Acervo Saúde / Electronic Journal Collection Health ISSN 2178-2091}

Tabela 1 - Avaliação dos juízes sobre os conteúdos das diretrizes de educação em saúde para profissionais de saúde que atuam no contexto do ensino médio calculado pelo índice de validade de conteúdo (1ª rodada método Delphi). Recife-PE, 2019.

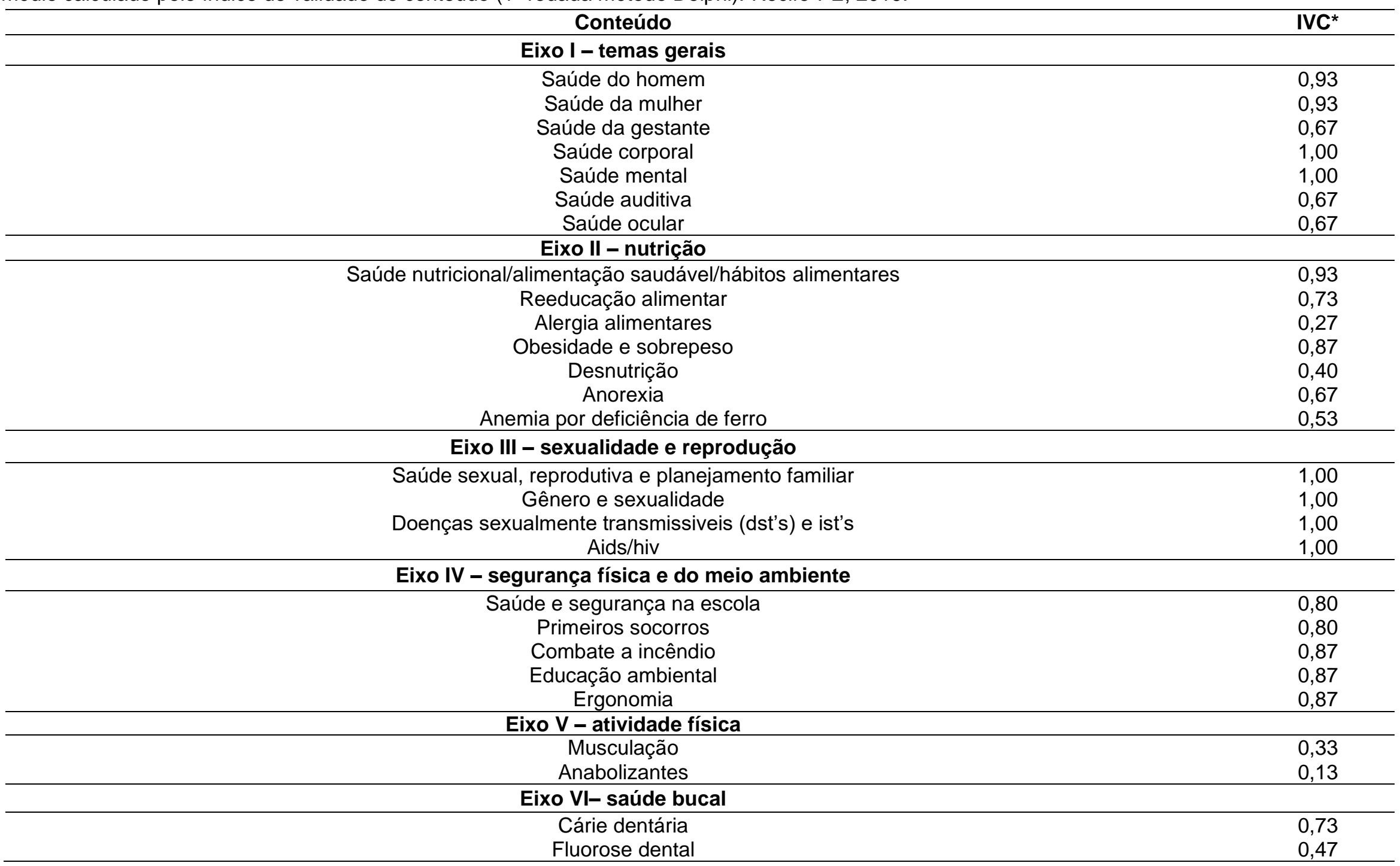

REAS/EJCH | Vol.12(6) | e3175 | DOI: https://doi.org/10.25248/reas.e3175.2020 Página 5 de 19 
Revista Eletrônica Acervo Saúde / Electronic Journal Collection Health ｜ ISSN 2178-2091

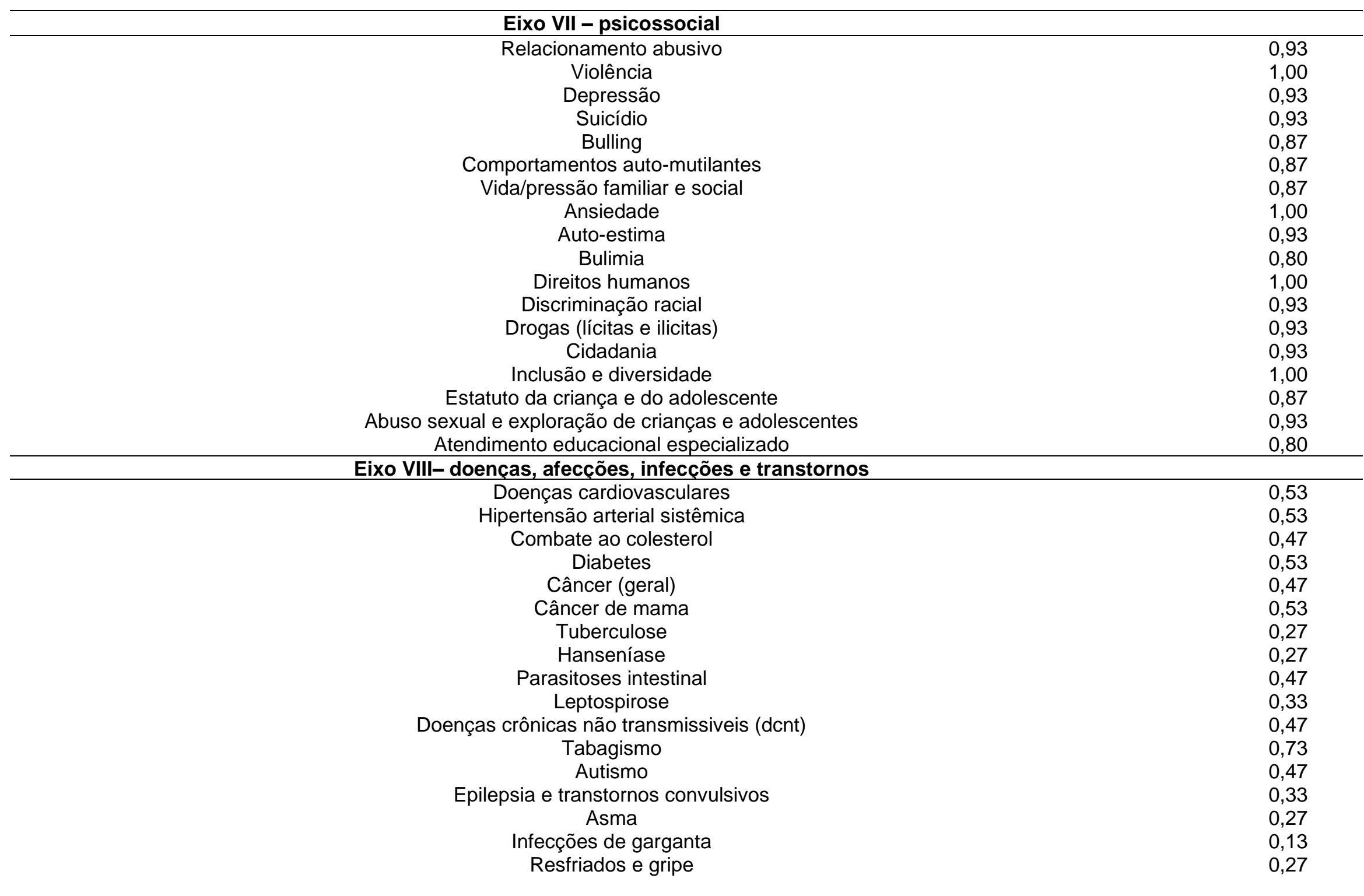

REAS/EJCH | Vol.12(6) | e3175 | DOI: https://doi.org/10.25248/reas.e3175.2020 Página 6 de 19 


\section{Revista Eletrônica Acervo Saúde / Electronic Journal Collection Health ｜ ISSN 2178-2091}

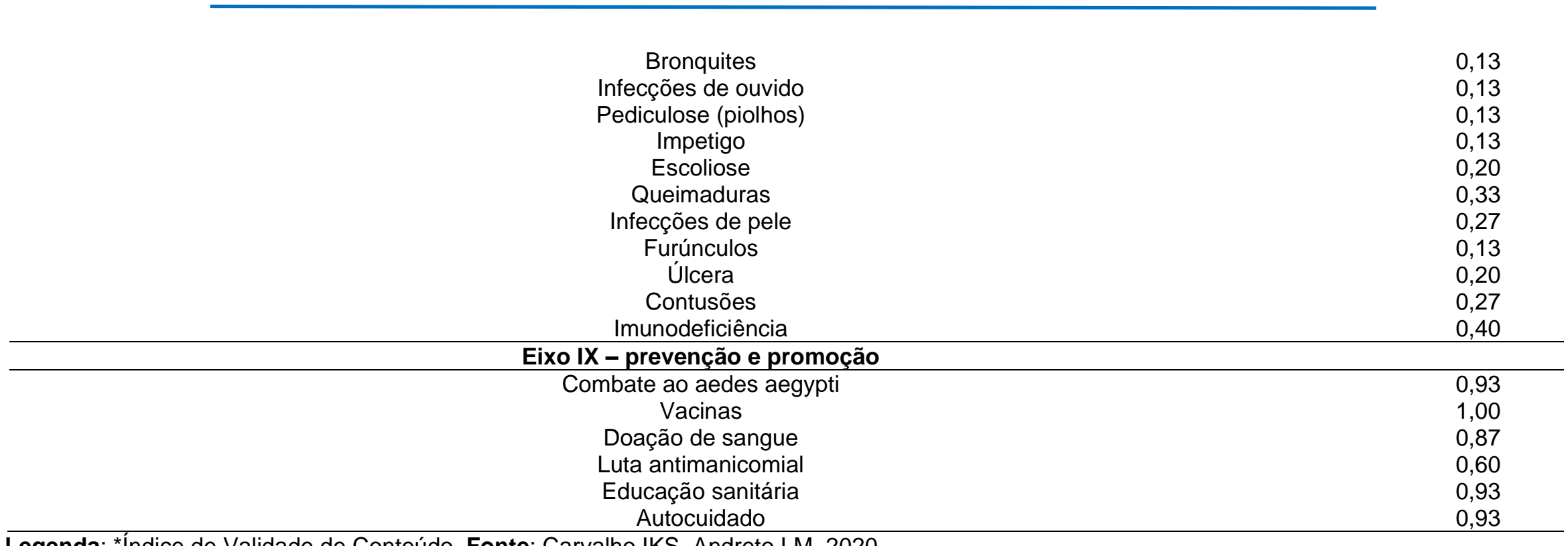




\section{Revista Eletrônica Acervo Saúde / Electronic Journal Collection Health | ISSN 2178-2091}

Tabela 2 - Avaliação dos juízes sobre o conteúdo das diretrizes de educação em saúde para profissionais de saúde que atuam no contexto do ensino médio calculado pelo índice de validade de conteúdo (2ª rodada método Delphi). Recife-PE, 2019.

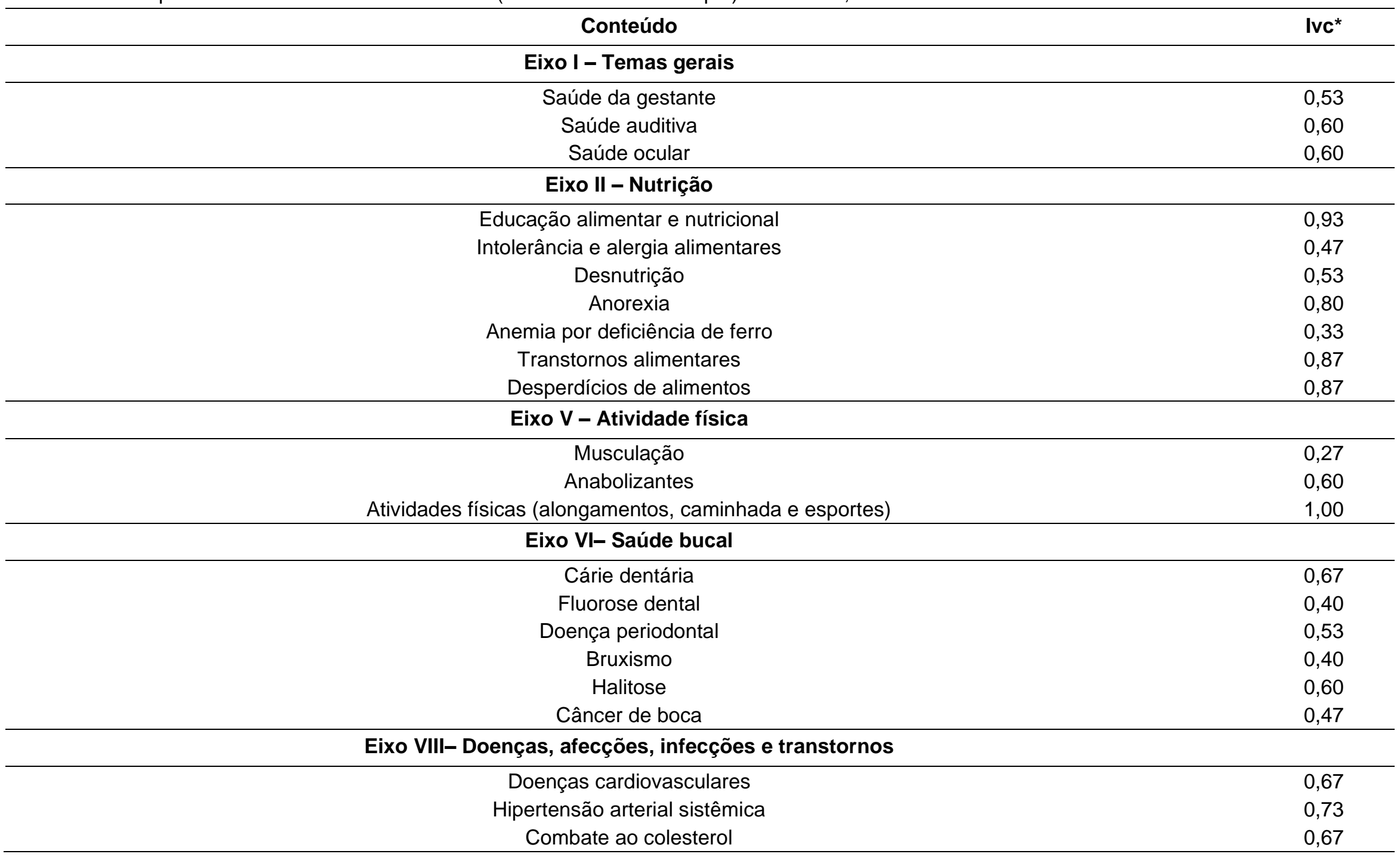

REAS/EJCH | Vol.12(6) | e3175 | DOI: https://doi.org/10.25248/reas.e3175.2020 Página 8 de 19 
Revista Eletrônica Acervo Saúde / Electronic Journal Collection Health ｜ ISSN 2178-2091

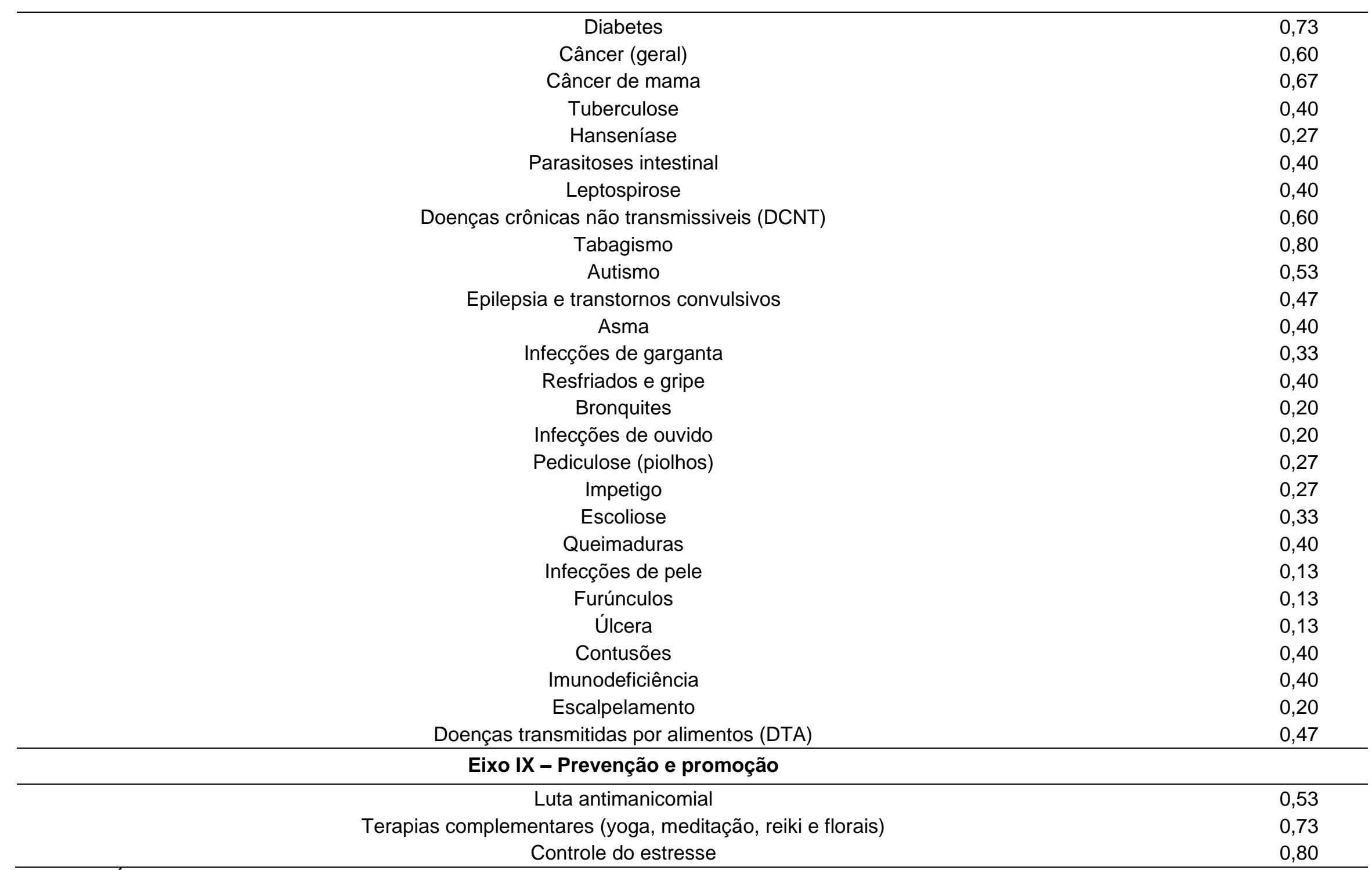

Legenda: *índice de Validade de Conteúdo. Fonte: Carvalho IKS, Andreto LM, 2020. 


\section{Revista Eletrônica Acervo Saúde / Electronic Journal Collection Health | ISSN 2178-2091}

Tabela 3 - Diretrizes de educação em saúde para profissionais de saúde que atuam no contexto do ensino médio validado pelos juízes especialistas - validação de conteúdo. Recife-PE, 2019.

\begin{tabular}{|c|c|}
\hline Conteúdo & IVC* \\
\hline \multicolumn{2}{|l|}{ Eixo I: Temas Gerais } \\
\hline Saúde do Homem & 0,93 \\
\hline Saúde da Mulher & 0,93 \\
\hline Saúde corporal & 1,00 \\
\hline Saúde Mental & 1,00 \\
\hline \multicolumn{2}{|l|}{ Eixo II: Nutrição } \\
\hline Saúde Nutricional/Alimentação saudável/Hábitos alimentares & 0,93 \\
\hline Educação alimentar e Nutricional & 0,93 \\
\hline Obesidade e Sobrepeso & 0,87 \\
\hline Anorexia & 0,80 \\
\hline Transtornos alimentares & 0,87 \\
\hline Desperdícios de alimentos & 0,87 \\
\hline \multicolumn{2}{|l|}{ Eixo III: Sexualidade e reprodução } \\
\hline Saúde Sexual, Reprodutiva e Planejamento Familiar & 1,00 \\
\hline Gênero e Sexualidade & 1,00 \\
\hline Doenças Sexualmente Transmissiveis (DST's) e IST's & 1,00 \\
\hline AIDS/HIV & 1,00 \\
\hline \multicolumn{2}{|l|}{ Eixo IV: Segurança física e do meio ambiente } \\
\hline Saúde e Segurança na Escola & 0,80 \\
\hline Primeiros Socorros & 0,80 \\
\hline Combate a incêndio & 0,87 \\
\hline Educação Ambiental & 0,87 \\
\hline Ergonomia & 0,87 \\
\hline \multicolumn{2}{|l|}{ Eixo V: Atividade física } \\
\hline Atividades físicas (alongamentos, caminhada e esportes) & 1,00 \\
\hline \multicolumn{2}{|l|}{ Eixo VI: Psicossocial } \\
\hline Relacionamento abusivo & 0,93 \\
\hline
\end{tabular}

REAS/EJCH | Vol.12(6) | e3175 | DOI: https://doi.org/10.25248/reas.e3175.2020 Página 10 de 19 


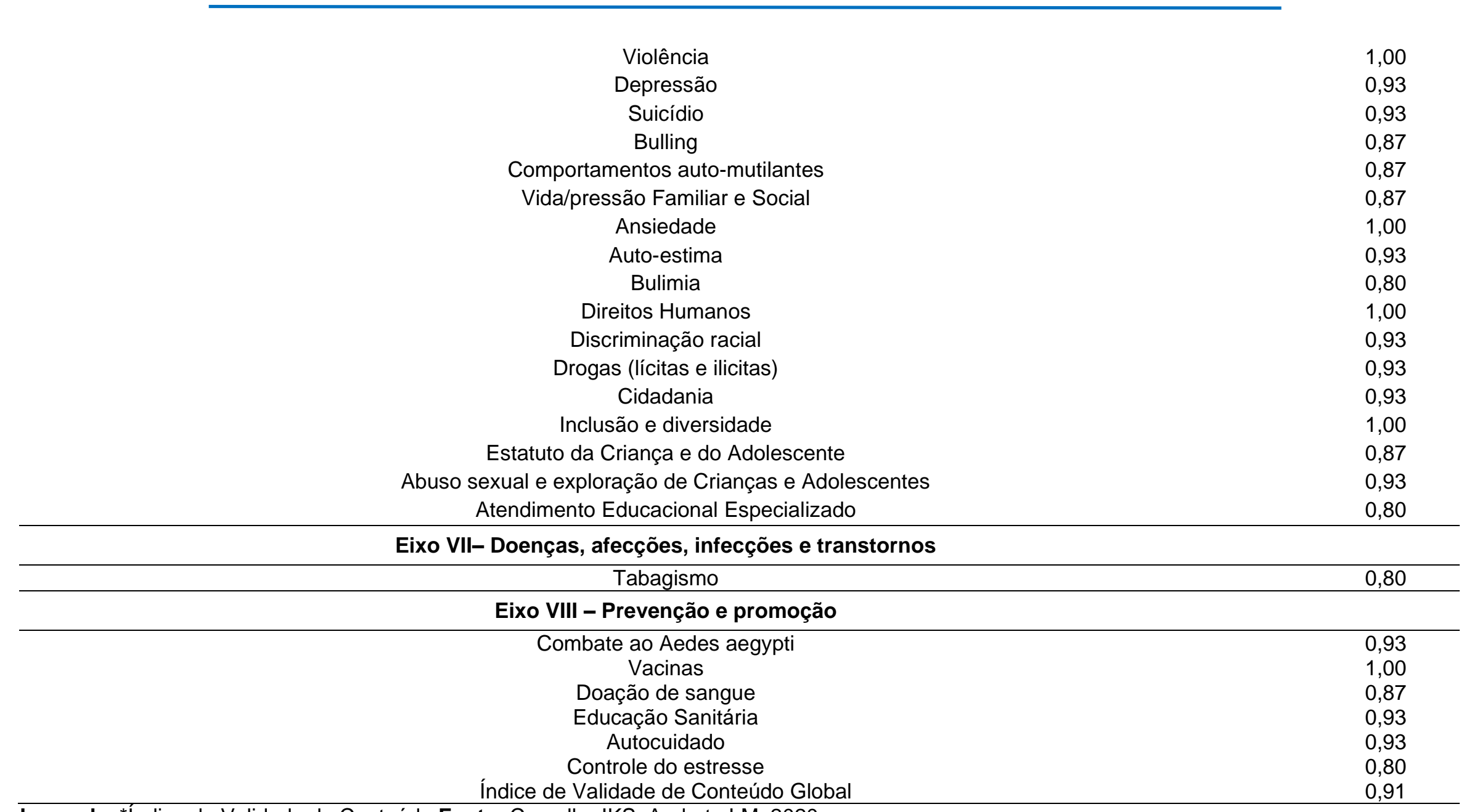

Legenda: *Índice de Validade de Conteúdo Fonte: Carvalho IKS, Andreto LM, 2020. 
Estes achados corroboram com os Parâmetros Curriculares Nacionais em Saúde que nesse contexto escolar traz a saúde sexual e reprodutiva como um tema de destaque. A higiene corporal é tratada nesse documento como condição para a vida saudável, a atividade física na puberdade e adolescência é apontada desempenhando papel importante não só do ponto de vista orgânico, mas psíquico também. A vacina, violência, diversidade e a saúde mental também são temas tratados e tidos como importantes nesse documento (MONT'ALVERNE DGB e CATRIB AMF, 2013).

O Programa Saúde na Escola atua na avaliação clínica, nutricional, oftalmológica, auditiva, psicossocial, da higiene bucal, promoção da alimentação saudável, atualização e controle do calendário vacinal, redução da morbimortalidade por acidentes e violências, prevenção e redução do consumo do álcool, prevenção do uso de drogas, promoção da saúde sexual e da saúde reprodutiva, controle do tabagismo e outros fatores de risco de câncer, educação permanente em saúde, atividade física e saúde, promoção da cultura da prevenção no âmbito escolar e inclusão das temáticas de educação em saúde no projeto político pedagógico das escolas (MONTEIRO PHI e BIZZO N, 2014). Essas temáticas acima corroboram parcialmente com o resultado desta pesquisa, uma vez que o eixo saúde bucal, assim como os itens saúde ocular, saúde auditiva e câncer não foram validados pelos juízes especialistas.

Estes resultados discordaram parcialmente de um estudo de revisão integrativa sobre a atuação de enfermeiros nas práticas de educação em saúde no contexto escolar que traz a saúde auditiva, saúde ocular e saúde da gestante como importantes práticas de saúde na escola. Já no que se refere ao Eixo II - Nutrição (que contempla os itens Saúde Nutricional/Alimentação saudável/Hábitos alimentares, Educação alimentar e Nutricional, Obesidade e Sobrepeso, Anorexia, Transtornos alimentares e Desperdícios de alimentos), Eixo III - Sexualidade e reprodução (que contempla os itens Saúde Sexual, Reprodutiva e Planejamento Familiar, Gênero e Sexualidade, Doenças Sexualmente Transmissiveis (DST's) e IST's e AIDS/HIV) e aos itens violência e drogas do Eixo VI - Psicossocial e tabagismo do Eixo VII - Doenças, afecções, infecções e transtornos, os resultados corroboraram (GUETERRES ÉC, et al., 2017).

Nesse mesmo estudo de revisão integrativa foi apresentado que $70 \%$ dos escolares apresentavam dentição comprometida. Este estudo supracitado salienta a importância do Eixo Saúde Bucal que não foi validado nesta pesquisa (GUETERRES ÉC, et al., 2017).

Um estudo relacionado aos cuidados de saúde na escola realizando comparações internacionais de casos na Austrália, Canadá e Estados Unidos da América apontaram basicamente as mesmas necessidades de saúde dos escolares: cuidados e rastreios de rotina, cuidados emergentes, doenças agudas, doenças crônicas, saúde mental, questões sociais, educação sanitária e necessidade de promoção (SEIGART D, et al., 2013).

Enfermeiros, professores e administradores nesses três países citados anteriormente relataram um aumento em crianças com condições crônicas, como asma, diabetes, autismo, distúrbios convulsivos e necessidades especiais relacionadas à deficiência de desenvolvimento. Eles também relataram doenças comuns, incluindo infecções na garganta, resfriados, gripes, bronquites, infecções de ouvido, infecções de pele, contusões, piolhos, impetigo, doenças sexualmente transmissíveis, necessidades de aconselhamento, necessidades de promoção da saúde e saúde bucal ruim (SEIGART D, et al., 2013).

Em relação a essas temáticas foram validados os itens saúde e segurança na escola, primeiros socorros, combate a incêndio, tabagismo, saúde mental, educação sanitária, além do Eixo VI - Psicossocial e Eixo VIII - Prevenção e promoção. Entretanto, houve, apenas, a validação do item tabagismo dentro do Eixo Doenças, afecções, infecções e transtorno discordando desse estudo que aponta a importância de temas relacionados a doenças crônicas e agudas nessa população (SEIGART D, et al., 2013).

Quando comparado a outro estudo americano, os resultados dessa pesquisa também divergiram em relação aos itens e temas como diabetes, asma, alergias alimentares e outras condições crônicas de saúde. Os itens diabetes, intolerância e alergias alimentares, e os itens do Eixo VII - Doenças, afecções, infecções e transtorno (com exceção do item tabagismo) não foram avaliados com IVC $\geq 0,80$ pelos juízes especialistas. Sendo assim, não foram validados (JONES SE, et al., 2015). 
Conforme exposto acima, temas considerados importantes por outros estudos científicos, não obtiveram a classificação como item indispensável ou muito importante para a educação em saúde no contexto escolar do Ensino Médio quando utilizado o ponto de corte de IVC $\geq 0,80$. É possível que o valor do ponto de corte recomendado pela literatura e utilizado pode ter influenciado no resultado, assim como a formação acadêmica e profissional dos participantes desse estudo. A visão e o entendimento da educação em saúde no contexto escolar do Brasil por parte dos profissionais de saúde que atuam nos Institutos Federais de Educação também pode ter influenciado nesse resultado (ALEXANDRE NM e COLUCI MZ, 2011).

No entanto, estudo de Alexandre NM e Coluci MZ (2011) aponta o ponto de corte mínimo de 0,80, recomendado na literatura em relação ao IVC para verificar a validade de novos instrumentos de uma forma geral.

O Fórum Nacional de Pró-Reitores de Assuntos Comunitários e Estudantis (FONAPRACE) - e a Associação Nacional de Pós-Graduandos (ANPG) têm sido atores políticos fundamentais para a construção de uma política de saúde para o estudante do ensino federal criando em 2012 durante o I Seminário de Atenção à Saúde do Estudante, um grupo de trabalho nacional de saúde do estudante com objetivo de discutir os princípios e diretrizes da assistência (BLEICHER T e OLIVEIRA RCN, 2016).

No relatório do FONAPRACE que trata do perfil de saúde dos estudantes de instituições federais foram identificados os seguintes temas de especial atenção para os estudantes: prevenção de doenças sexualmente transmissíveis, necessidade de intervenções em saúde mental, dependência química e programas de prevenção em saúde oral. Com a exceção do tema saúde bucal que não foi validado pelos juízes, os resultados dessa pesquisa corroboram com o relatório supracitado (BLEICHER T e OLIVEIRA RCN, 2016).

A validação semântica do conteúdo foi realizada por nove profissionais de saúde do IFSertão-PE. Quanto ao perfil acadêmico e profissional $88,9 \%$ (8) dos profissionais de saúde foram do sexo feminino, a idade média foi de 38,4 anos $\pm 9,5$ anos. Quanto a formação acadêmica, 33,3\% (3) são formados em Enfermagem, 22,2\% (2) em Psicologia, 22,2\% (2) em Serviço Social, 11,1\% (1) em Nutrição e 11,1\% (1) em Medicina, com tempo de experiência profissional com a média de 13,4 anos. Todos possuíam pós-graduação, $66,7 \%$ (6) possuíam Mestrado Stricto Sensu.

A Tabela 4 apresenta os 45 itens que obtiveram a validação de conteúdo pelos juízes especialistas no tema na $2^{\circ}$ etapa deste estudo e mostra o resultado da primeira rodada do método Delphi quanto a análise semântica realizada pelos profissionais de saúde (população-alvo).

Nesta etapa, 42 itens apresentaram índice de validade com concordância maior ou igual a $0,80($ IVC $\geq 0,80)$ e três tiveram IVC $<0,80$. Os itens que não obtiveram consenso na primeira rodada foram: saúde corporal com IVC=0,67, saúde e segurança na escola com IVC=0,78, e Educação ambiental com IVC=0,78.

$\mathrm{Na}$ segunda rodada foi solicitado aos profissionais de saúde para reescrever o item conforme seu entendimento e embasamento científico ou reavaliar novamente sua resposta da primeira rodada, do item que não obteve consenso, caso quisesse reconsiderar sua avaliação. 


\section{Revista Eletrônica Acervo Saúde / Electronic Journal Collection Health ｜ ISSN 2178-2091}

Tabela 4 - Avaliação dos profissionais de saúde (população-alvo) sobre os aspectos semânticos das diretrizes de educação em saúde para profissionais de saúde que atuam no contexto do ensino médio calculado pelo índice de validade de conteúdo (1 ${ }^{a}$ rodada método Delphi). Recife-PE, 2019.

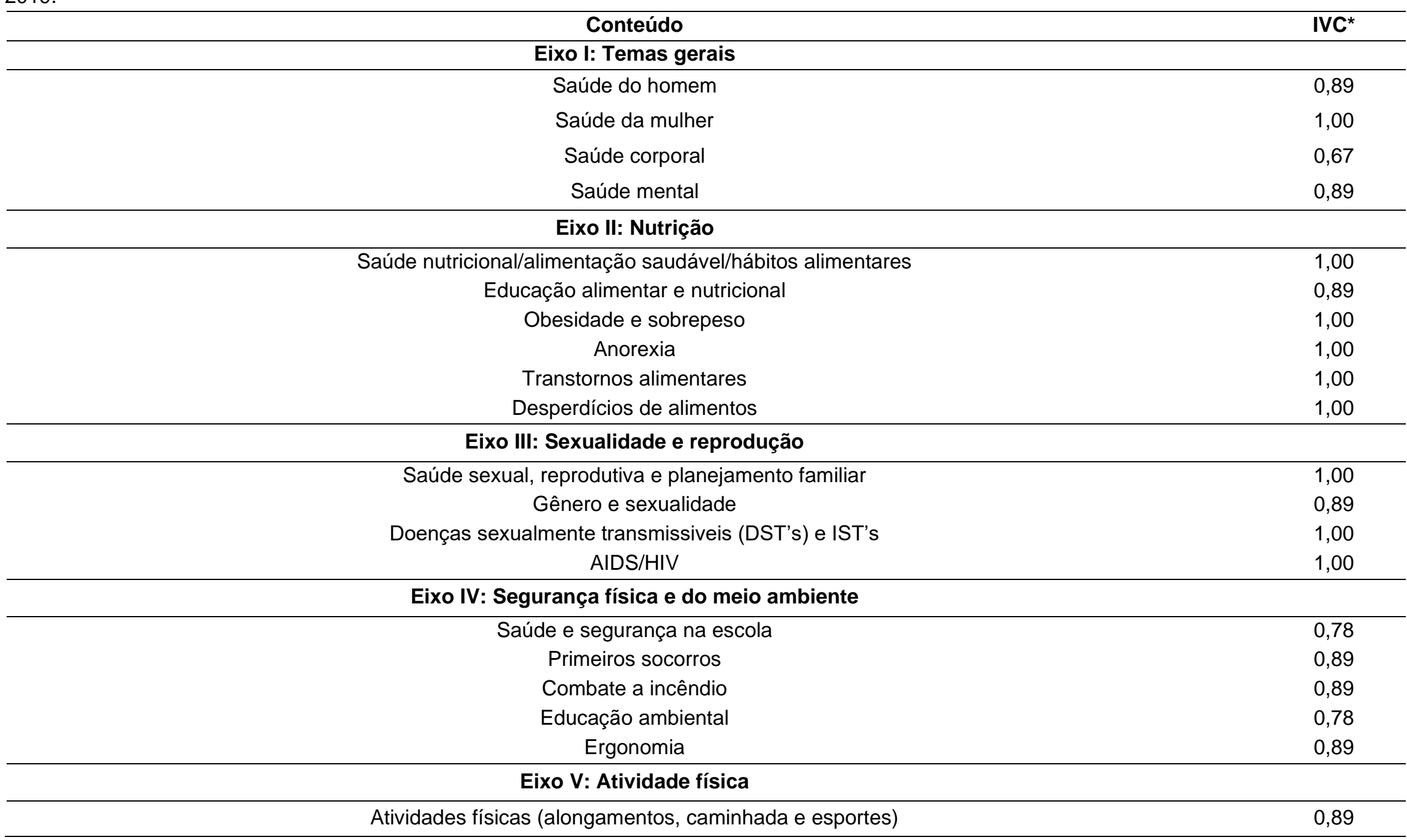

REAS/EJCH | Vol.12(6) | e3175 | DOI: https://doi.org/10.25248/reas.e3175.2020 Página 14 de 19 
Revista Eletrônica Acervo Saúde / Electronic Journal Collection Health ｜ ISSN 2178-2091

\begin{tabular}{|c|c|}
\hline Eixo VI: Psicossocial & \\
\hline Relacionamento abusivo & 0,89 \\
\hline Violência & 1,00 \\
\hline Depressão & 1,00 \\
\hline Suicídio & 1,00 \\
\hline Bulling & 1,00 \\
\hline Comportamentos auto-mutilantes & 1,00 \\
\hline Vida/pressão familiar e social & 1,00 \\
\hline Ansiedade & 1,00 \\
\hline Auto-estima & 1,00 \\
\hline Bulimia & 1,00 \\
\hline Direitos humanos & 1,00 \\
\hline Discriminação racial & 1,00 \\
\hline Drogas (lícitas e ilicitas) & 1,00 \\
\hline Cidadania & 1,00 \\
\hline Inclusão e diversidade & 1,00 \\
\hline Estatuto da criança e do adolescente & 1,00 \\
\hline Abuso sexual e exploração de crianças e adolescentes & 1,00 \\
\hline Atendimento educacional especializado & 0,89 \\
\hline \multicolumn{2}{|l|}{ Eixo VII - Doenças, afecções, infecções e transtornos } \\
\hline Tabagismo & 1,00 \\
\hline \multicolumn{2}{|l|}{ Eixo VIII - Prevenção e promoção } \\
\hline Combate ao Aedes aegypti & 1,00 \\
\hline Vacinas & 1,00 \\
\hline Doação de sangue & 1,00 \\
\hline Educação sanitária & 1,00 \\
\hline Autocuidado & 0,89 \\
\hline Controle do estresse & 1,00 \\
\hline
\end{tabular}

Legenda: *índice de Validade de Conteúdo. Fonte: Carvalho IKS, Andreto LM, 2020. 
Tabela 5 - Abaixo, mostra-se o resultado da segunda rodada que teve a participação de cinco dos nove juízes participantes da primeira rodada, sendo assim, nesta rodada, tive-se a perda de $45,5 \%$ (4) dos profissionais de saúde do grupo inicial selecionado para realizar a validação semântica.

\begin{tabular}{cc}
\hline & IVC $^{*}$ \\
\hline Eixo I: Temas gerais & \\
\hline Saúde corporal & 0,60 \\
Higiene corporal (em substituição ao item saúde corporal) & 0,40 \\
\hline Eixo IV: Segurança física e do meio ambiente & 1,00 \\
\hline Saúde e segurança na escola & 1,00 \\
\hline
\end{tabular}

Legenda: *índice de validade de conteúdo. Fonte: Carvalho IKS, Andreto LM, 2020.

Observa-se, na Tabela 5, o IVC=1,00 para os itens Educação ambiental e Saúde e segurança na escola que foram reavaliados e não tiveram sugestões de reescrita, porém 0 item saúde corporal não obteve consenso novamente tendo o IVC=0,60. O termo Higiene corporal foi incluído para avaliação dos juízes por estar presente nos Parâmetros Curriculares Saúde, sendo posto em substituição ao item saúde corporal, mas também não obteve consenso, tendo o IVC $=0,40$. Os juízes não apresentaram sugestão de reescrita para o item saúde corporal.

Dessa forma optou-se, de forma análoga ao estudo de Santos FC (2016), por manter a escrita dos itens educação ambiental, saúde e segurança na escola e saúde corporal no instrumento, mesmo sem o consenso deste último, pois não foram apontadas outras sugestões de reescrita para o item saúde corporal. A Tabela 6 apresenta os conteúdos validados semanticamente e seus respectivos Índices de Validade de Conteúdo, assim como o IVC Global. 


\section{Revista Eletrônica Acervo Saúde / Electronic Journal Collection Health ISSN 2178-2091}

Tabela 6 - Diretrizes de educação em saúde para profissionais de saúde no contexto do ensino médio calculado pelo índice de validade de conteúdo - validação semântica. Recife-PE, 2019.

\begin{tabular}{|c|c|}
\hline Conteúdo & IVC* \\
\hline \multicolumn{2}{|l|}{ Eixo I: Temas gerais } \\
\hline Saúde do homem & 0,89 \\
\hline Saúde da mulher & 1,00 \\
\hline Saúde corporal & 0,60 \\
\hline Saúde mental & 0,89 \\
\hline \multicolumn{2}{|l|}{ Eixo II: Nutrição } \\
\hline Saúde nutricional/alimentação saudável/hábitos alimentares & 1,00 \\
\hline Educação alimentar e nutricional & 0,89 \\
\hline Obesidade e sobrepeso & 1,00 \\
\hline Anorexia & 1,00 \\
\hline Transtornos alimentares & 1,00 \\
\hline Desperdícios de alimentos & 1,00 \\
\hline \multicolumn{2}{|l|}{ Eixo III: Sexualidade e reprodução } \\
\hline Saúde sexual, reprodutiva e planejamento familiar & 1,00 \\
\hline Gênero e sexualidade & 0,89 \\
\hline Doenças sexualmente transmissiveis (DST's) e IST's & 1,00 \\
\hline AIDS/HIV & 1,00 \\
\hline \multicolumn{2}{|l|}{ Eixo IV: Segurança física e do meio ambiente } \\
\hline Saúde e segurança na escola & 1,00 \\
\hline Primeiros socorros & 0,89 \\
\hline Combate a incêndio & 0,89 \\
\hline Educação ambiental & 1,00 \\
\hline Ergonomia & 0,89 \\
\hline \multicolumn{2}{|l|}{ Eixo V: Atividade física } \\
\hline Atividades físicas (alongamentos, caminhada e esportes) & 0,89 \\
\hline
\end{tabular}

REAS/EJCH | Vol.12(6) | e3175 | DOI: https://doi.org/10.25248/reas.e3175.2020 Página 17 de 19 
Revista Eletrônica Acervo Saúde / Electronic Journal Collection Health ～ISSN 2178-2091

\begin{tabular}{|c|c|}
\hline \multicolumn{2}{|l|}{ Eixo VI: Psicossocial } \\
\hline Relacionamento abusivo & 0,89 \\
\hline Violência & 1,00 \\
\hline Depressão & 1,00 \\
\hline Suicídio & 1,00 \\
\hline Bulling & 1,00 \\
\hline Comportamentos auto-mutilantes & 1,00 \\
\hline Vida/pressão familiar e social & 1,00 \\
\hline Ansiedade & 1,00 \\
\hline Auto-estima & 1,00 \\
\hline Bulimia & 1,00 \\
\hline Direitos humanos & 1,00 \\
\hline Discriminação racial & 1,00 \\
\hline Drogas (lícitas e ilicitas) & 1,00 \\
\hline Cidadania & 1,00 \\
\hline Inclusão e diversidade & 1,00 \\
\hline Estatuto da criança e do adolescente & 1,00 \\
\hline Abuso sexual e exploração de crianças e adolescentes & 1,00 \\
\hline Atendimento educacional especializado & 0,89 \\
\hline \multicolumn{2}{|l|}{ Eixo VII - Doenças, afecções, infecções e transtornos } \\
\hline Tabagismo & 1,00 \\
\hline \multicolumn{2}{|l|}{ Eixo VIII - Prevenção e promoção } \\
\hline Combate ao Aedes aegypti & 1,00 \\
\hline Vacinas & 1,00 \\
\hline Doação de sangue & 1,00 \\
\hline Educação sanitaria & 1,00 \\
\hline Autocuidado & 0,89 \\
\hline Controle do estresse & 1,00 \\
\hline Índice de Validade de Conteúdo Global & 0,96 \\
\hline
\end{tabular}

Legenda: *Índice de Validade de Conteúdo Fonte: Carvalho IKS, Andreto LM, 2020. 


\section{CONCLUSÃO}

Este estudo elaborou e validou os conteúdos das diretrizes de Educação em Saúde para o ensino médio que obteve um IVC Global de 0,91 em relação à validação de conteúdo e um IVC Global de 0,96 em relação a validação semântica. Desse modo, o produto desse estudo consiste em um instrumento contendo 45 itens distribuídos em oito eixos que poderá ser utilizado como instrumento de orientação aos profissionais de saúde dos Institutos Federais de Educação.

\section{REFERÊNCIAS}

1. ALEXANDRE NM, COLUCI MZ. Validade de conteúdo nos processos de construção e adaptação de instrumentos de medidas. Ciênc. Saúde coletiva, v 16, n. 7, PP 3061-8, 2011.

2. BLEICHER T, OLIVEIRA, RCN. Políticas de assistência estudantil em saúde nos institutos e universidades federais. Psicologia Escolar e Educacional. 2016; 20(3), 543-549.

3. BOND, KS, et al. Development of guidelines for family and non-professional helpers on assisting an older person who is developing cognitive impairment or has dementia: a Delphi expert consensus study. BMC Geriatrics, July $7^{\text {th }}$ 2016, Vol.16(1), pp.129.

4. BRASIL. Conselho Nacional de Saúde. Resolução n 466, de 12 de Dezembro de 2012. Diário Oficial da União 13 jun 2012.

5. BRASIL. Decreto ํㅜ 6.286, de 05 de Dezembro de 2007. Institui o Programa Saúde na Escola - PSE, e dá outras providências. Diário Oficial da União 06 dez 2007.

6. BRASIL. Decreto № 7.234, de 19 de Julho de 2010. Dispõe sobre o Programa Nacional de Assistência Estudantil PNAES. Diário Oficial da União 20 jul 2010.

7. CARDOSO V, et al. Escolas Promotoras de Saúde. Rev Bras Crescimento Desenvol Hum. 2008; 18(2): $107-115$.

8. GUETERRES ÉC, et al. Educación para la salud en el contexto escolar: estudio de revisión integradora. Enferm. glob. [Internet]. 2017

9. INSTITUIÇÃO. In: Instituto Federal de Educação, Ciência e Tecnologia Sertão Pernambucano. Disponível em: http:www.ifsertao-pe.edu.br. Acesso em: 11 out. 2019.

10. JONES SE, et al. Association Between School District Policies that Address Chronic Health Conditions of Students and Professional Development for School Nurses on such Policies. The Journal of school nursing: the official publication of the National Association of School Nurses. 2015;31(3): 163-166.doi:10.1177/1059840514547275.

11. LIKERT RA. Technique for the measurement of attitudes. Archives of Psychology. n. 140, p. 44-53, 1932.

12. MCISAAC JLD, et al. Interventions to Support System-level Implementation of Health Promoting Schools: A Scoping Review. International Journal of Environmental Research and Public Health. 2016; 13(2):200.

13. MINISTÉRIO DA EDUCAÇÃO. Secretaria de Educação Fundamental. Parâmetros curriculares nacionais: introdução aos parâmetros curriculares nacionais. Brasília (DF); 1997.

14. MONT'ALVERNE DGB, CATRIB AMF. Promoção da saúde e as escolas: como avançar. Rev Bras em Promoção da Saúde. 2013;26(3): 307-308.

15. MONTEIRO PHI, BIZZO N. A saúde na escola: análise dos documentos de referência nos quarenta anos de obrigatoriedade dos programas de saúde, His Cienc Saúde. 2014;22(2): 411-427.

16. PARÂMETROS CURRICULARES NACIONAIS SAÚDE. 1997. In: Ministério da Educação. Disponível em: http://portal.mec.gov.br/seb/arquivos/pdf/saude.pdf. Acesso em: 27 out. 2019.

17. PILATTI L, et al. Propriedades psicométricas de instrumentos de avaliação: Um debate necessário. Rev. Bras. Ensino Ciência Tecnol., v. 3, n. 1, PP. 81-91, 2010.

18. ROGERS R, et al. Top 10 Lessons Learned from Project Healthy Schools. The American Journal of Medicine. 2017; Volume 130, Issue 8, 990.e1 - 990.e7.

19. SANTOS FC. Construção e Validação Semântica de um instrumento para avaliação de competências de enfermeiros que atuam em oncologia [dissertação]. Ribeirão Preto: Universidade de São Paulo, Escola de Enfermagem de Ribeirão Preto; 2016.

20. SEIGART D, et al. Barriers to providing school-based health care: International case comparisons. Collegian. 2013; Volume 20, Issue 1, $43-50$. 